\title{
Sensibilidad a antimicrobianos comunes de bacterias obtenidas en urocultivos positivos de niños
}

\author{
Claudia Giglio M. ; Carlos Toro A. ${ }^{2}$ \\ Antimicrobial susceptibility of bacteria \\ from positive urine cultures
}

\begin{abstract}
Antimicrobial susceptibility of 400 consecutive positive bacterial isolates from urifle cultures $120^{5}$ coicny counts per ml) of a simiiar number of ambulatory $\{n \cdot 216)$ and hospital ( $n$ : 184 ) male and female pa:ients $\{300$ girls) under age 15 year, from downtown Santiago. Chile, is described. All samples were processed at a university associated hospital of the chilean public health services system. Bacterial sensitivity was tested by disk aiffusion (Kirby-Bauert. Escherichia colf was by far the most frequently isolated pathogenic bacteria $: 75.7 \%$ of the whole, $89.1 \%$ outpatient girls, $58.5 \%$ outpatier.t bovs, $76.8 \%$ inoatient emales and $45.7 \%$ incatient malesi. Klebsielta pneumoniae was second in outpatients girls (3.4\%), and Proteus mirabilis in boys (22.0\%). Among iripatients Kieb. sizlia pneumoniae, plus Kiebsjella sp were second $\{13.4 \%$ ir gir.s and $18.5 \%$ in males). Pseudomonas aeruginosa was only identified from inpatient males $\{10.1 \%$. One third of $E$. coli comming from ambulatory patients were resistant to first generation cephalosporins, more than $50 \%$ were not sensitive to ampicillin and trimethoprim. sulfamethoxazole. but $96 \%$ or more wiere susceptible to gentamicin and a similar proporion to nitrofurantoin i> $96 \%)$. In samples obtained irom rospital patients, $28 \%$ E. coli were resistant to the arrpicillin-sulbactam association (p 0.0000053 ), more than $40 \%$ to firs: neneration ceonlosporins, over $50 \%$ to trimethoprim-sulfamethoxazole and a similar percentage to ampicillin. wh ile $95 \%$ or more were susceptibie tc amikacin and genta`ricin to 0.000 . K. pneumoniae were almost always sensitive 10 amikacin (93./\%) but gentamicin was efective ir. only 20\% of thein (p 0.000148). Almost one half $K$. pneumoniae were sensitive to nitrofuranzoin as well. Six in seven cases of $P$. aeruginoss were susceptible to cettazidime. Unless local susceptibility studies show diferent results it looks reasonable to sugest that, in this comunity, initiat treatment of patients under age 1 b var with lower urinary tract infections should include seriousconsideration to nitrofurantoin as a first line drug. The same seems to be true for amikacin, while susceptibility tests are worked up, when upper urinary infections are suspecterd among these patients.
\end{abstract}

(Key words: Urifary infection, antírricrooial, susceotibility.)

1. Residente Becario de Pediatría. Departamento de Pediatría, Cirugía Infantil y Ortopedia, División Central, Facultad de Medicina, Universidad de Chile. Hospital San Borja-Arriarán.
2. Profesor Asociado de Pediatría. Departamento de Pediatría, Cirugía Infantil y Ortopedia, División Central, Facultad de Medicina, Universioad de Chile. Hospital \$an Borja-Arriarân. 
La infección de la via urinaria sigue constituyendo una enfermedad importante en pediatría, no sólo por su frecuencia, sino por el riesgo potencial que involucra. Su diagnóstico oportuno, correcto manejo $y$ adecuado seguimiento permiten disminuir la incidencia de daños renales posteriores. El tratamiento apropiado de estos pacientes incluye, entre sus pilares fundamentales, el uso racional de diversas drogas antimicrobianas.

La búsqueda de información sobre la fre. cuencia relativa de aislamientos de los diferentes microorganismos que suelen causar infecciones del tracto urinario en niños y su sensibilidad a los antibióticos de empleo habitual. en una base de datos conocida (Lilacs, CD-ROM años 1982 a 1991), mostró que ellas no han sido motivo de publicaciones en las revistas de mayor circulación en los últimos đjez años, no obstante lo cual se han reactualizado recientemente recomendaciones de grupos de expertos nacionales para su tratamiento'. Este estudio se realizó con los propósitos de describir la frecuencia relativa con que se aíslan los diferentes agentes bacterianos de infección de la vía urinaria, en los urocultivos de pacientes pediátricos -hospitalizados y ambulatorios- que son procesados en un hospital general metropolitano, así como su susceptibilidad a los antimicrobianos de uso común y establecer si las recomendaciones más recientes sobre la materia ofrecen bases racionales en la elección de la terapia inicial con antibióticos en pacientes con infecciones urinarias.

\section{Material y Método}

Para los fines de este estudio se recuperaron de modo prospectivo y semanalmente, de los libros de registro del laboratorio de microbiología del Hospital Clínico San Borja-Anjarán, todos los resultados de los urocultivos de pacientes menores de 15 años de edad, cuyos recuentos de colonias fuesen iguales o mayores de $10^{5}$ colonjas por $\mathrm{nl}^{2}$, que proviniesen de los consultorios y servicios hospitalarios del Servicio de Salud Metropolitano Central de Santiago y tuviesen información suficiente sobre edad, sexo y procedencia del paciente. La recolección de datos se hizo de manera continua, programada y sucesiva a partir del 1 de octubre de 1990 , incluyendo todas las muestras que cumpliesen los requisitos señalados hasta completar un total de 400 . Los estudios de sensibilidad a los antibióticos se realizaron mediante pruebas de difusión en agar ${ }^{3}$, utilizando discos para antibiograma con ácido pipemídico $(20 \mu \mathrm{g})$, amikacina $(30 \mu \mathrm{g})$, ampicilina (10 $\mu \mathrm{g}$ ), ampicilina-sulbactam (10 y $10 \mu \mathrm{g}$ ), cefotaxima (30 $\mu \mathrm{g})$, cefalotina $(30 \mu \mathrm{g})$ (tomando esta última como modelo de sensibilidad de las cefalosporinas de primera generación ${ }^{4}$, ciprofloxacino $(5 \mu \mathrm{g}$ ), cotrimoxazol (sulfametoxazol $23.75 \mu \mathrm{g}$ y trimetropim $1,25 \mu \mathrm{g}$ ), gentamicina $(10 \mu \mathrm{g})$ y nitrofurantóna $(300 \mu \mathrm{g})$ para las muestras provenientes de pacientes hospitalizados. En los ensayos de sensibilidad de aislamientos obtenidos de niños ambulatorios se usó ácido nalidíxico (30 $\mu \mathrm{g}$ ), ácido pipemídico, ampicilina, cefalotina, cotrimoxazol, gentamicina y nitrofurantoína. Cuando se aisló Pseudomono aerzginosa, Staphylococcus, u otros gémenes menos corrientes, se utilizaron discos especiales, de acuerdo a la sensibilidad descrita en otras experiencias ${ }^{5}$. los que se identifican en el momento de expresar los resultados, cuando es oporturo. Las diferencias en la seleccion de discos para las muestras de procedencia ambulatoria y hospitalaria obedece a razones de planificación de las acciones de salud del servicio correspondiente, a la experiencia previa del laboratorio, al coste y disponibilidad de los recursos. El diámetro de la zona de inhibición usado para interpretar la sensibitidad fue el mismo que ha recomendado en normas más recientes el "Centro Nacional pasa Control de Calidad de Estándares de Laboratorios de los EUA' (SCCLS vol II, No 17 , december 1991) y su validez en comparación con las pruebas de concentración inhi. bitoria mínima parece suficientemente asentada ${ }^{4}$. Cuando el número de ajslamientos lo permitjó, se comparó la sensibilidad de cada germen a los diferentes antibióticos, mediante el método de $\chi^{2}$ y la prueba cxacta de Fisher, estableciendo en $p<0,001$ el nivel de significación para las diterencias encontradas.

\section{Resultados}

Doscientas dieciséis de las 400 muestras pro. venian de pacientes ambulatorios $(54 \%)$ y 300 $(75 \%)$ eran de niñas. Independientemente de la procedencia y el sexo, Escherichia coli (E. coll) fue el germen más frecuentemente aislado $(75,7 \%)$ en todo el conjunto de muestras. En las muestras ambulatorias la frecuencia de $E$, coli en mujeres fue $89 \%$ y $58 \%$ en hombres; $K l e b$ siella pneumoniae fue el segundo germen más frecuente en las mujeres y Proteus mirabilis en los hombres, mientras Klebsiella pneumoniae y Klebsiella sp fueron segundas en frecuencia en pacientes hospitalizados de los dos sexos. Cabe destacar que no se identificó Pseudomona aeruginosa -aislada predominantemente de varones hospitalizados- y Morganella marganii de mues. tras de pacientes ambulatorios. La distribución de los agentes identificados en pacientes hospitalizados mostró también una tendencia similar en lo que respecta a $E$. coll, pero la de los demás gérmenes fue ligeramente diferente (tabla 1).

La sensibilidad de las diferentes bacterias patógenas aisladas de las 216 muestras de pacientes 
Tabla I

Frecuencia relativa de los diferenies agentes aisłados en 400 cultivos positivos de niños ambulatorios y hospitalizados, según sexo y procedencia

\begin{tabular}{|c|c|c|c|c|c|c|c|c|c|c|}
\hline & \multicolumn{4}{|c|}{ Ambulatorios } & \multicolumn{4}{|c|}{ Hospitalizados } & \multicolumn{2}{|c|}{ Total } \\
\hline & \multicolumn{2}{|c|}{ Mujeres } & \multicolumn{2}{|c|}{ Hombres } & \multicolumn{2}{|c|}{ Mujeres } & \multicolumn{2}{|c|}{ Hombres } & \multirow[b]{2}{*}{$\mathrm{n}$} & \multirow[b]{2}{*}{$\%$} \\
\hline & $\mathfrak{n}$ & 4 & $\mathrm{n}$ & $\%$ & $\mathrm{n}$ & $\%$ & $\mathrm{n}$ & $\%$ & & \\
\hline E. coli & 156 & 89,1 & 24 & 58,5 & 96 & 76,8 & 27 & 45,7 & 303 & 75,5 \\
\hline K. pneumaniae & 6 & 3,4 & - & - & 11 & 8.8 & 5 & 8,4 & 22 & 5,5 \\
\hline Klebsiella $s p$. & 1 & 0,5 & 3 & 7,3 & 7 & 5,6 & 6 & 10,1 & 17 & 4,2 \\
\hline P. mirabilis & 1 & 0,5 & 9 & 22.0 & 3 & 2.4 & 3 & 5,0 & 16 & 4,0 \\
\hline P. aeruginasa & - & - & - & - & 2 & 1,6 & 6 & 10,1 & 8 & 2,0 \\
\hline P. bulgaris & - & - & 2 & 4,9 & 1 & 0,8 & 2 & 3,3 & 5 & 1,2 \\
\hline C. freundij & 2 & 1,1 & 1 & 2,4 & 1 & 0,8 & - & - & 4 & 1,0 \\
\hline S. oxytoca & 2 & 1,1 & 1 & 2,4 & - & - & 1 & 1,6 & 4 & 1,0 \\
\hline Enterobacter sp. & 2 & 1,1 & - & - & I & 0,8 & 1 & 1,6 & 4 & 1,0 \\
\hline M. morganii & - & - & - & - & 1 & 0,8 & 2 & 3,3 & 3 & 0,7 \\
\hline S. epidermis & 1 & 0,5 & 1 & 2,4 & I & 0,8 & - & - & 3 & 0,7 \\
\hline S. aureus & l & 0.5 & - & - & - & - & 2 & 3,3 & 3 & 0,7 \\
\hline E. aglomerans & 1 & 0.5 & - & - & - & - & 1 & 1,6 & 2 & 0,5 \\
\hline S. soprophyticus & - & - & - & .. & 1 & 0.8 & 1 & 1,6 & 2 & 0,5 \\
\hline C. diverstus & 1 & 0,5 & - & - & - & - & 1 & 1,6 & 2 & 0,5 \\
\hline Citrohacter sp. & - & - & - & - & - & - & 1 & 1,6 & 1 & 0,2 \\
\hline Hafina alvei & l & 0,5 & - & - & - & - & - & - & 1 & 0,2 \\
\hline Total & 175 & 100,0 & 41 & 100,0 & 125 & 100,0 & 59 & 100,0 & 400 & 100,0 \\
\hline
\end{tabular}

ambulatorios se describe en la tabla 2, donde se puede observar que la mayoría ( $96 \%$ o más) de las cepas de $E$. coll eran sensibles a ácido nalidíxico, gentamicina, ácido pipemídico y nitrofurantóna, mientras que más de la mitad eran resistentes a ampicilina y cotrimoxazol y un tercio era resistente a las cefalosporinas de primera generación $(\hat{p} 0,0000)$. La menor sensibilidad a ampicilina y cotrimoxazol es tambièn estadísticamente significativa con respecto a la observada para las cefalosporinas de primera generaciớn.

En pacientes hospitalizados (tabia 3), la mayoría (95\% o más) de las cepas de $E$. coli eran susceptibles a amikacina, gentamicina, acido pipemídico, nitrofurantoina, cefotaxima y ciproflo. xacino, pero sólo $72 \%$ a ampicilina-sulbactam. La resistencia a la aludida combinación es estadisticamente significativa (p 0,0000053 ). Cuarenta por ciento de $E$. coli de muestras obteni. das en el hospital eran resistentes a cefalotina, más de la mitad a cotrimoxazol y ampicilina. La falta de sensibilidad a estos tres últimos me. dicamentos fue estadísticamente similar entre ellos, aunque no necesariamente simultánea, $y$, en todos los casos, significativamente mayor que para todos los medicamentos precedentemente citados. Sólo 3 de 123 cepas de $E$. coll ensayadas eran sensibles a ceftazidima. Cabe destacar que $93,7 \%$ de Klebsiella preumoniae eran sensibles a amikacina, pero sólo $20 \%$ a gentamicina (p 0,0001480), diferencia que no ocurrió en el caso de $E$. coli. Aunque el escaso número de cepas de Psetudomona aeruginosa no permite un análisis apropiado de significación, la mayoría $(80 \%)$ eran susceptibles a ceftazidima y ciprofloxacino y más de la mitad a los aminoglucósidos, pero todas eran resistentes a ampicilina, ampicilina-sulbactam, nitrofurantoina y cefalotina. 
Tabla 3

Sensihilidad a los antibióticos de bacterias más frecuentemente aisladas de urocultivos positivos en niños hospitalizados

Ac.pjpemid. Arrikacina Ampicilina Amp.-sulb, Cefalotina Cefotaxima Ceftazidima Ciprofloxa Cotrimoxazol Gentamicina Nitrofur.

\begin{tabular}{|c|c|c|c|c|c|c|c|c|c|c|c|c|c|c|c|c|c|c|c|c|c|c|}
\hline & $\mathbf{n}$ & $\%$ & $n$ & $\%$ & $\mathrm{n}$ & $\%$ & $n$ & $\%$ & $\mathrm{n}$ & $\psi_{0}$ & n & $\gamma_{0}$ & $\mathbf{n}$ & $\%$ & $\mathbf{n}$ & $\%$ & $\mathrm{n}$ & $\%$ & $\mathbf{n}$ & $\%$ & $\mathrm{n}$ & $\%$ \\
\hline E. coll & $\begin{array}{l}115 \\
(120\end{array}$ & 95,8 & $\begin{array}{l}119 \\
(119)\end{array}$ & 100,0 & $\begin{array}{l}28 \\
(121)\end{array}$ & 23,1 & $\begin{array}{l}78 \\
(108\end{array}$ & $\begin{array}{l}72,2 \\
\text { ) }\end{array}$ & $\begin{array}{l}35 \\
(58\end{array}$ & 60,3 & $\begin{array}{l}99 \\
110\end{array}$ & $\begin{array}{l}99,0 \\
0)\end{array}$ & $\begin{array}{l}3 \\
(12\end{array}$ & 2,4 & $\begin{array}{l}52 \\
(52)\end{array}$ & 100,0 & $\begin{array}{l}57 \\
(122\end{array}$ & 46,7 & $\begin{array}{l}119 \\
(122\end{array}$ & 97,5 & $\begin{array}{l}115 \\
(121\end{array}$ & $\begin{array}{l}95,0 \\
1)\end{array}$ \\
\hline K. pneumoniae & $\begin{array}{l}8 \\
(16)\end{array}$ & 50,0 & $\begin{array}{l}13 \\
(16)\end{array}$ & 93.7 & $\begin{array}{l}0 \\
(16)\end{array}$ & 0,0 & $\begin{array}{l}0 \\
(15)\end{array}$ & 0,0 & $\begin{array}{l}1 \\
(9)\end{array}$ & 11,1 & $\begin{array}{l}4 \\
(14\end{array}$ & 28,5 & $\begin{array}{l}1 \\
\text { (4) }\end{array}$ & 25,0 & $\begin{array}{l}3 \\
\text { (4) }\end{array}$ & 75,0 & $\begin{array}{l}0 \\
(16)\end{array}$ & 0,0 & $\begin{array}{l}3 \\
(15)\end{array}$ & 20,0 & $\begin{array}{l}7 \\
(16)\end{array}$ & 43,7 \\
\hline Klebsiella sp. & $\begin{array}{l}11 \\
(13)\end{array}$ & 84,6 & $\begin{array}{l}12 \\
(13)\end{array}$ & 92,3 & $\begin{array}{l}2 \\
(13)\end{array}$ & 15,3 & $\begin{array}{l}2 \\
\text { (13) }\end{array}$ & 15,3 & $\begin{array}{l}1 \\
(1)\end{array}$ & 100,0 & $\begin{array}{l}9 \\
(10\end{array}$ & 90,0 & - & - & $\begin{array}{l}3 \\
(3)\end{array}$ & 100,0 & $\begin{array}{l}3 \\
(13)\end{array}$ & 23,0 & $\begin{array}{l}5 \\
(13)\end{array}$ & 38,4 & $\begin{array}{l}13 \\
\text { (13) }\end{array}$ & 100,0 \\
\hline P. mirubilis & $\begin{array}{l}4 \\
\text { (6) }\end{array}$ & 66,6 & $\begin{array}{l}6 \\
(6)\end{array}$ & 100,0 & $\begin{array}{l}3 \\
\text { (6) }\end{array}$ & 50,0 & $\begin{array}{l}3 \\
(5)\end{array}$ & 60,0 & $\begin{array}{l}4 \\
\text { (6) }\end{array}$ & 66.6 & $\begin{array}{l}4 \\
(4)\end{array}$ & 100,0 & $\begin{array}{l}- \\
-\end{array}$ & - & $\begin{array}{l}2 \\
\text { (2) }\end{array}$ & 100,0 & $\begin{array}{l}3 \\
\text { (6) }\end{array}$ & 50,0 & $\begin{array}{l}6 \\
(6)\end{array}$ & 100,0 & $\begin{array}{l}0 \\
(6)\end{array}$ & 0,0 \\
\hline$P$ aeruginos $a$ & $\begin{array}{l}4 \\
(5)\end{array}$ & 80,0 & $\begin{array}{l}5 \\
(8)\end{array}$ & 62,5 & $\begin{array}{l}0 \\
(8)\end{array}$ & 0,0 & $\begin{array}{l}0 \\
(8)\end{array}$ & 0,0 & $\begin{array}{l}0 \\
(5)\end{array}$ & 0,0 & $\begin{array}{l}1 \\
(7)\end{array}$ & 14,2 & $\begin{array}{l}6 \\
(7)\end{array}$ & 85,7 & $\begin{array}{l}6 \\
(7)\end{array}$ & 85,7 & $\begin{array}{l}0 \\
(7)\end{array}$ & 0,0 & $\begin{array}{l}5 \\
(8)\end{array}$ & 62,5 & $\begin{array}{l}0 \\
(5)\end{array}$ & 0,0 \\
\hline C. fresindii & $\begin{array}{l}1 \\
(1)\end{array}$ & 100,0 & $\begin{array}{l}1 \\
\text { (1) }\end{array}$ & 100,0 & $\begin{array}{l}0 \\
(1)\end{array}$ & 0,0 & $\begin{array}{l}0 \\
\text { (1) }\end{array}$ & 0,0 & $\begin{array}{l}- \\
-\end{array}$ & & $\begin{array}{l}1 \\
\text { (1) }\end{array}$ & 100,0 & $\begin{array}{l}- \\
-\end{array}$ & - & $\begin{array}{l}- \\
-\end{array}$ & - & $\begin{array}{l}- \\
-\end{array}$ & - & $\begin{array}{l}\mathrm{l} \\
(1)\end{array}$ & 100,0 & - & - \\
\hline K. axytaca & $\begin{array}{l}1 \\
(1)\end{array}$ & 100,0 & $\begin{array}{l}1 \\
(1)\end{array}$ & 100,0 & $\begin{array}{l}0 \\
(1)\end{array}$ & 0,0 & $\begin{array}{l}0 \\
01 \mathrm{j}\end{array}$ & 0,0 & $\begin{array}{l}- \\
-\end{array}$ & & $\begin{array}{l}1 \\
(1)\end{array}$ & 100,0 & $\begin{array}{l}- \\
-\end{array}$ & - & $\begin{array}{l}- \\
-\end{array}$ & - & $\begin{array}{l}0 \\
(1)\end{array}$ & 0,0 & $\begin{array}{l}1 \\
(1)\end{array}$ & 100,0 & $\begin{array}{l}1 \\
(1)\end{array}$ & 100,0 \\
\hline Enterobacter sp. & $\begin{array}{l}2 \\
(2)\end{array}$ & 100.0 & $\begin{array}{l}1 \\
(2)\end{array}$ & 50,0 & $\begin{array}{l}0 \\
(2)\end{array}$ & 0,0 & $\begin{array}{l}0 \\
(2)\end{array}$ & 0,0 & $\begin{array}{l}0 \\
(1)\end{array}$ & 0,0 & $\begin{array}{l}2 \\
(2)\end{array}$ & 100,0 & - & - & $\begin{array}{l}1 \\
(2)\end{array}$ & 50,0 & $\begin{array}{l}1 \\
(2)\end{array}$ & 50,0 & $\begin{array}{l}0 \\
(2)\end{array}$ & 0,0 & $\begin{array}{l}1 \\
(2)\end{array}$ & 50,0 \\
\hline
\end{tabular}

Entre paréntesis el múmero de cepas en que se stalizó la prucba correspondiente. 


\section{Comentario}

Escherichia coli sigue siendo el germen más frecuentemente aislado de urocultivos positivos en niños, al menos en el ámbito del Servicio de Salud Metropolitano Central, particularmente en mujeres, donde su predoninio es más acentuado que en varones, en quienes, a la inversa, la proporción de los agentes secundarios es mayor que en las niñas, lo que sugiere grados diferentes de contaminación $y$ diferentes tipos de problemas clínicos, posibilidad que se ve respaldada por el hecho de aparecer diferencias por sexo entre los gérmenes menos frecuentes, en favor de Klebsiella pneumoniae en mujeres $y$ Proteus mirabilis en hombres, asi como Pseudomona aeruginosa, que sólo se aisló de hospitalizados, preferentemente en varones. Todo to anterior no inplica necesariamente que estas frecuencias relativas sean idénticas a las que se encontrarían en pacientes que cumpliesen criterios clínicos más estrictos de infección urinatia, lo que excedería las pretenciones de este estudio dedicado, por definición, al análisis de urocultivos positivos, con desarrollo de números significativos de colonias, lo que, sin embargo, no invalida los resultados de la susceptibilidad de los agentes aislados. Las cepas de Escherichia coll obtenidas de muestras provenientes tanto de consultorios come del hospital resultaron sensibles a numerosos antibióticos, pero algunos de ellos no pueden ser recomendados, dada la rápida adquisición de resistencia que caracteriza a su uso, como es el caso de los ácidos nalidíxico y pipemidico. La nitrofurantoina sigue siendo una opción altamente recomendable para el tratamiento de infecciones de la vía urinaria baja, por su efectividad, amplio espectro, bajo coste y fácil administración. En los pacientes con evidencia de infección del parénquirna renal parece razonable recomendar que se emplee inicialmente, mientras se elabora el estudio de sensibilidad, gentamicina o amikacina, especialmente la última, en vista de su mayor efectividad sobre Klebsiella pnewmoniae en pacientes hospitalizados de esta serie $y$ en otras experiencias $^{6}$. Los efectos de nefrotoxicidad de los aminoglucósidos se pueden evitat empleando dosis e intervalos adecuados para cada paciente, controlando la creatininemia al ingreso, al tercer día $y$ al final del tratamiento en los casos en que los antecedentes del paciente lo justifiquen $y$, finalmente, cambiando de medicamento, cuando esto sea posible y oportuno, si así lo recomiendan la respuesta clinica y el informe de sensibilidad $\mathbf{I}, 5$

La recomendación reciente de utilizar cefalosporinas de primera generación o cotrimoxazol en el tratamiento inicial de los niños con infecciones urinarias ${ }^{1}$ debe ser -a nuestro juicioreconsiderada a la luz de estos resultados, pues la tasa relativamente alta de resistencia de las cepas de $E$. coli a estos medicamentos sugiere, al menos, cautela en el momento de elegirlos sin antibiograma previo, lo que también podía ser válido para quimioprofilaxis con cotrimoxazol, a menos que estudios locales similares a éste demuestren una situación diferente con respecto a ellos, tomando en cuenta no sólo la posibilidad de fracaso, sino también la de generar rápida e innecesariamente mayor resistencia, especialmente a las cefalosporinas, que la que existe actualmente, tal como ha ocurrido evidentemente con ampicilina. La información periódica de los laboratorios a los médicos clínicos, sobre la susceptibjlidad bacteriana a los antibióticos, asume aquí toda su importancia. Como se ha señalado en el método, el empleo de técnicas de difusión de disco no resta validez a esta manera de enfrentar el problema de la resistencia, si su interpretación es la apropiada ${ }^{4}$ y se tienen en cuenta las diferencias entre sensibilidad in vitro y respuesta en vivo. La sensibilidad a cefalosporinas de tercera generación estaba más allá de las posibilidades de esta investigación -orientada a antibióticos de uso común en nuestros hospitales públicos-, ya que, si bien son muy útiles, su elevado coste y el riesgo de producir creciente resistencia, invalidándolas como medicamentos de reserva, nos inducen a sugerir que deben guardarse, idealmente, para situaciones que no dejan otras opciones.

Con respecto a ciprofloxacina, quinolona a la cual las cepas identificadas mostraron alta sensibilidad, su uso ha sido dejado momentáneamente de lado sobre la base de evidencias experimentales, en cachorros de perro, de daño secundatio en el cartilago de crecimiento. Sin embargo, el medicamento ha sido aplicado a niños con fjbrosis quística, sin que ellos registrasen posteriormente problemas osteoarticulares $^{7}$. 


\section{Resumen}

Se describe la frecuencia relativa de las bacterias patógenas y su sensibilidad a los antimicrobianos en 400 urocultivos sucesivos con recuentos de colonias $\geqslant 10^{5}$ de personas de 150 menos años de edad, ambulatorias (n: 216) y hospitalizadas, de ambos sexos (300 niñas). Escherichia coli fue el agente aislado con mayor frecuencia $(75,7 \%$ del total, $89,1 \%$ en niñas y $58,5 \%$ en varones ambulatorios, $76,8 \%$ en niñas y 45,7 en niños hospitalizados), seguido de Klebsiella pneumoniae en niñas $(3,5 \%)$ y Proteus mirabilis en varones $(22,0 \%)$ ambulatorios. En pacientes del hospital, Klebsiella pneumoniae y Klebsiella species fueron segundas en frecuencia en niñas $(13,4 \%)$ y varones $(18,5 \%)$. Pseudomonas aenaginosa sólo se detectó en pacientes del hospital $(10,1 \%$ varones, $1,6 \%$ niffas $)$. Un tercio de las cepas de $E$. coli eran resistentes a cefalosporinas de primera generación, más de la mitad a ampicilina y cotrimoxazol, siendo la mayoria $(\geqslant 96 \%$ ) sensibles a gentamicina $y$ nitrofurantoína. En las muestras de niños hospitalizados, $28 \%$ E. coli eran resistentes a ampicilina-sulbactam (p 0,0000053), más de 40\% a cefalosporinas de primera generación y más de $50 \%$ a cotrimoxazol y ampicilina, mientras $95 \%$ o más eran sensibles a amikacina $y$ gentamicina (p 0,000$). K$. pneumoniae fueron casi siempre sensibles a amikacina $(93,7 \%)$. pero sólo $20 \%$ a gentamicina ( 0,000148 ), siendo alrededor de la mitad sensibles a nitrofurantoína. Seis de siete cepas de Pseudomonas aeruginosa etan sensibles a ceftazidima. A menos que estudios locales muestren una situación diferente, parece razona. ble sugerir que pacientes ambulatorios con infecciones urinarias aparentemente bajas sean tratados inicialmente de preferencia con nitro. furantoína y se prefiera amikacina si se sospe. cha infección alta, modificando posteriormente la conducta, si es conveniente, según el análisis de sensibilidad.

(Palabras claves: Infección urinatia, microbiología, antibjograma.)

\section{Referencias}

1. Rodriguez $E$, Vargas $B$, Olivares $\beta$ et al.: Infección del tracto urinario. Rev Chil Pediatr 1990: 61 (Supl. 1): 14-16.

2. Kass EH: Asymptomatic infections of the urinay tract. Trans Assoc Am Physicians 1956; 69: 56.

3. Barry AL. Thomsberry C: Susceptibitity Test: Diffusion Test Procedures. In: Ballows A. Manual of Clinical Microbiolngy, Washington DC: American Society for Microbiology, Fifth edition 1991: $1117-1125$

4. Washington $f$ : How the microbiology laboratory can improve antimicsobial therapy. Bull NY Acad Med 1984: 60: 314-326.

5. Anónimo: Elección de medicamentos antimicro. bianos. Carta Médica, Ministerio de Salud de Chile, abril $1991: 40-54$

6. Goodman $A$ y Gilman T. Goodman GA, editor: Las bases farmacológicas de la terapéutica, Octaya edición. Buenos Aires, Editorial Panamericana 1991, p. 1028.

7. Neu H: Quinolones a new class of antimicrobial agents with wide potential uses. Med Clin North Am 1988; 72: 623-636.

\section{AVISO A LOS AUTORES}

Por acuerdo del Comité Editorial, la Revista Chilena de Pediatría devolverá sin tramitar todos los trabajos que no den estricto cumplimiento al Reglamento de Publicaciones y a las Instrucciones a los Autores que se edjtan en cada número de la Revista. 